\title{
Near-infrared Fluorescence Imaging and Optimization of Polyacrylamide
}

\author{
Yan Chen ${ }^{1, a}$, Jinke Wang ${ }^{2, b,{ }^{*}}$ \\ 1,2 State Key Laboratory of Bioelectronics, School of Biological Sciences and Medical Engineering, \\ Southeast University, Nanjing 210096, China
}

a220151652@seu.edu.cn, bwangjinke@seu.edu.cn

Keywords: Polyacrylamide, Fluorescence, Effect Factors.

\begin{abstract}
Polyacrylamide (PAA) is a reticular macromolecular compound, and the $\mathrm{C}=\mathrm{N}$ bonds produced by the reaction with glutaraldehyde reveal intensive near-infrared fluorescence (NIRF). This paper studies the effect factors on the fluorescence intensity of PAA microspheres (beads), including the particle size of PAA beads, APMA concentrations, and different concentrations of glycine treatment by infrared imaging system and fluorescence microscope. The results demonstrate that PAA gel and PAA beads display autofluorescence at the emission wavelength of $700 \mathrm{~nm}$ and 800 $\mathrm{nm}$, respectively. The smaller the particle size of beads is, the better the fluorescence intensity is in a certain range. The beads have stronger NIRF when $11 \mathrm{mg} / \mathrm{mL}$ APMA is in monomer liquid compared with the beads containing $7 \mathrm{mg} / \mathrm{mL}$ or $9 \mathrm{mg} / \mathrm{mL}$ APMA in monomer liquid. Glycine treatment enhances the fluorescence intensity of the beads, which is the most obvious at $0.2 \mathrm{M}$ glycine in solution.
\end{abstract}

\section{Introduction}

Polyacrylamide (PAA) gel is a macromolecule polymerization of acrylamide and $\mathrm{N}$, N'-Methylene-Bis-Acrylamide (bisacrylamide) under the action of cross-linking agent. PAA is widely applied to thickening [1], reducing drag [2], adsorption [3], flocculation [4], biomedical materials and so on for many excellent advantages, including good flocculation, low cost, easy preparation. In biomedical research, PAA materials are prepared in different forms based on various needs and have a wide range of applications. For instance, PAA gel is often used in the separation and purification of nucleic acid and proteins. PAA hydrogel has good application prospects in plastic surgery [5] and drug release because of its excellent biological compatibility, innocuity and environmental friendliness. PAA nanoparticles (PAANP) have huge application potential in drug loading [6] and tumor near-infrared fluorescence imaging [7]. PAA microspheres (PAA beads) have been applied to SNP genotyping [8] in previous studies.

Glutaraldehyde is a kind of straight chain aliphatic dialdehyde with each aldehyde group in position 1 and 5, which can react with various chemical groups respectively. Therefore, glutaraldehyde has been widely used in cell protein immobilization [9] and the crosslinking of biomaterials [10]. It has been reported that the $\mathrm{C}=\mathrm{N}$ bonds on Schiff base which produced by the reaction between amino groups and aldehyde groups display autofluorescence [11]. Since the amino groups on acrylamide and bisacrylamide are rather inert, N-(3-Aminopropyl)methacrylamide hydrochloride (APMA) is employed as the comonomer to carry out the copolymerization [12] and increase the active amino groups. Glycine is a common amino acid and is often used in biochemical experiments and organic synthesis. The amino groups on glycine can react with the aldehyde groups on glutaraldehyde to produce $\mathrm{C}=\mathrm{N}$ bonds, which is utilized to enhance the fluorescence intensity of the polyacrylamide in this study.

This paper studies the preparation method of fluorescent polyacrylamide materials and researches the effects of the particle size of PAA beads, APMA concentrations and different concentrations of glycine treatment on the fluorescence intensity. 


\section{Materials and Methods}

\subsection{The preparation of fluorescent PAA gel in 384 well plates and fluorescent PAA beads}

Polyacrylamide monomer liquid: $264 \mathrm{mg}$ Acrylamide, $80 \mathrm{mg} \mathrm{N}$, N'-Methylene-Bis-Acrylamide, 7 mg N-(3-Aminopropyl)methacrylamide hydrochloride (APMA), $1 \mathrm{~mL} \mathrm{H2O}$, dissolution under ultrasound at $37{ }^{\circ} \mathrm{C}$. The gel solutions contained $1 \mathrm{~mL}$ monomer liquid, $985 \mu \mathrm{L} \mathrm{H} 2 \mathrm{O}, 14 \mu \mathrm{L}$ Ammonium Persulphate (APS) and $1 \mu \mathrm{L}$ N,N,N', N'-Tetramethylethylenediamine (TEMED). The gel solutions were pipetted $20 \mu \mathrm{L}$ into each well of 384 well plates. The gel was polymerized at $37{ }^{\circ} \mathrm{C}$ for $1 \mathrm{~min}$. Add $20 \mu \mathrm{L}$ glutaraldehyde ( $5 \%$ ) each well into 3 wells and $20 \mu \mathrm{L} \mathrm{H} 2 \mathrm{O}$ each well into another 3 wells as a control. The reaction was at $37{ }^{\circ} \mathrm{C}$ overnight.

Prepare the same monomer liquid as above. The mixed solutions contained $335 \mu \mathrm{L}$ monomer liquid, $50 \mu \mathrm{L}$ sodium borate buffer $(0.2 \mathrm{M}), 20 \mu \mathrm{L}$ APS, $595 \mu \mathrm{L} \mathrm{H} 2 \mathrm{O}$. The mineral oil containing $0.4 \%$ TEMED was placed into a plate with polyethylene, and $1.0 \mu \mathrm{L}$ aliquots of the mixed solution were pipetted under the mineral oil. Beads were polymerized at $37{ }^{\circ} \mathrm{C}$ for $1 \mathrm{~min}$. The mineral oil was decanted and the beads were recovered in $\mathrm{H} 2 \mathrm{O}$. Half of these beads were put into $1 \mathrm{~mL}$ glutaraldehyde $(5 \%)$ and the other half were put into $1 \mathrm{~mL} \mathrm{H} 2 \mathrm{O}$ as a control. The reaction was at $37{ }^{\circ} \mathrm{C}$ overnight.

\subsection{Different effect factors of the fluorescence intensity of the beads}

The particle size of PAA beads: Make the beads as above. $1.0 \mu \mathrm{L}, 0.5 \mu \mathrm{L}$ and $0.25 \mu \mathrm{L}$ aliquots of the mixed solution were pipetted under the mineral oil for making different particle sizes of beads. Put these three kinds of beads into different $1.5 \mathrm{~mL}$ centrifuge tubes and add $1 \mathrm{~mL}$ glutaraldehyde $(5 \%)$ separately. The reaction was at $37{ }^{\circ} \mathrm{C}$ overnight.

APMA concentrations: Prepare 3 kinds of different monomer liquids, containing $7 \mathrm{mg}, 9 \mathrm{mg}, 11$ mg APMA separately. Make the beads as above. Put these three kinds of beads into different $1.5 \mathrm{~mL}$ centrifuge tubes and add $1 \mathrm{~mL}$ glutaraldehyde (5\%) separately. The reaction was at $37{ }^{\circ} \mathrm{C}$ overnight.

Different concentrations of glycine treatment: Make the beads as above and the monomer liquid contains $11 \mathrm{mg}$ APMA. Use $5 \%$ glutaraldehyde to react with the beads at $37{ }^{\circ} \mathrm{C}$ overnight and wash them with water. Then these beads were soaked in $1 \mathrm{~mL} 0 \mathrm{M}, 0.1 \mathrm{M}, 0.2 \mathrm{M}, 0.3 \mathrm{M}$ glycine solutions separately at $37^{\circ} \mathrm{C}$ for $6 \mathrm{~h}$.

\section{Results}

\subsection{NIRF of glutaraldehyde-treated PAA gel and PAA beads}

Glutaraldehyde-treated PAA gel was imaged with various fluorescence intensity by using Odyssey Infrared Imaging System and the results indicated that the glutaraldehyde-treated PAA gel showed autofluorescence at the emission wavelength of both $700 \mathrm{~nm}$ and $800 \mathrm{~nm}$ while PAA itself did not emit fluorescence (Fig.1a).

PAA beads after treated by glutaraldehyde were also imaged under various fluorescence intensity and the results of NIRF revealed that the glutaraldehyde-treated PAA beads showed autofluorescence at the emission wavelength of both $700 \mathrm{~nm}$ and $800 \mathrm{~nm}$ but PAA itself did not emit fluorescence and the fluorescence at $700 \mathrm{~nm}$ was stronger than that at $800 \mathrm{~nm}$ (Fig.1b).

\subsection{The effect factors of the fluorescence intensity of PAA beads}

The imagings by the fluorescence microscope show that different particle sizes of glutaraldehyde-treated PAA beads all had intensive green, red and blue fluorescence (Fig.2a). NIRF imaging results suggest the smaller the particle size of beads is, the stronger the fluorescence intensity is in a certain range (Fig. $2 b$ ).

The NIRF scanning results demonstrate that the beads containing $11 \mathrm{mg} / \mathrm{mL}$ APMA in monomer liquid had stronger fluorescence no matter at the emission wavelength of $700 \mathrm{~nm}$ or $800 \mathrm{~nm}$ 


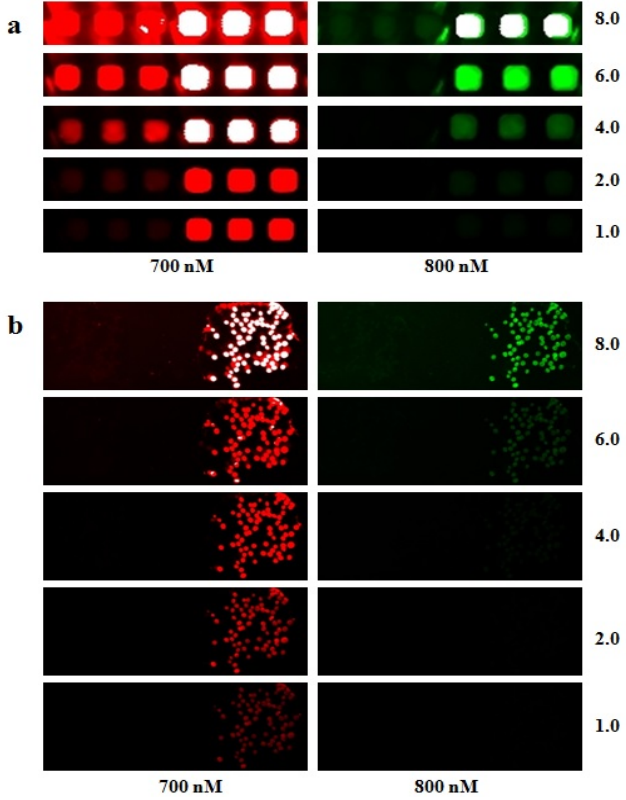

Fig. 1. Near infrared fluorescence (NIRF) of glutaraldehyde-treated PAA. (a) NIRF of glutaraldehyde-treated PAA gel. (b) NIRF of glutaraldehyde-treated PAA beads. a
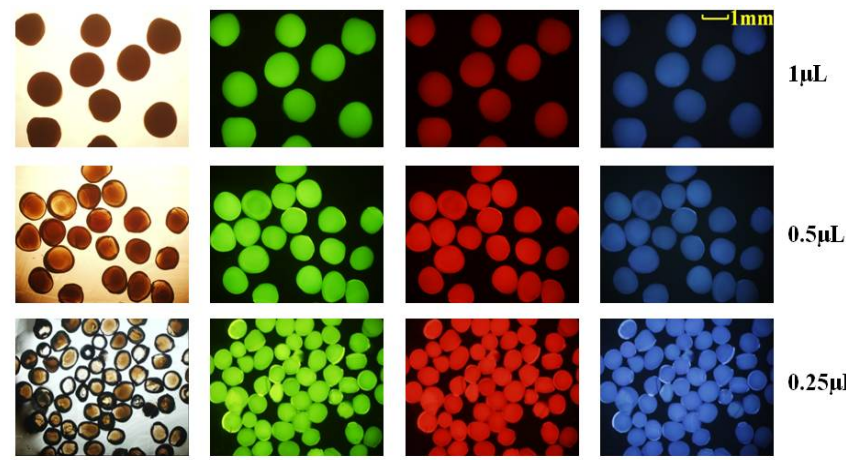

$1 \mu \mathrm{L}$
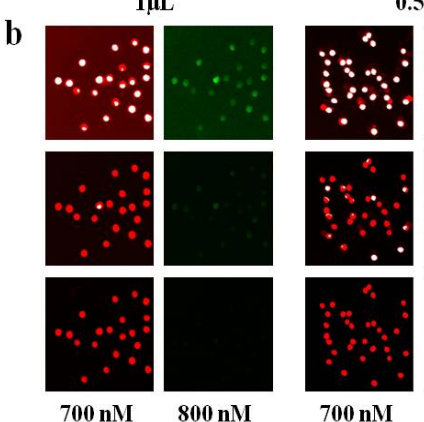

$0.5 \mu \mathrm{L}$
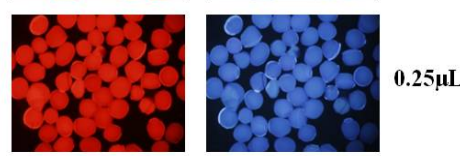

$0.25 \mathrm{uL}$
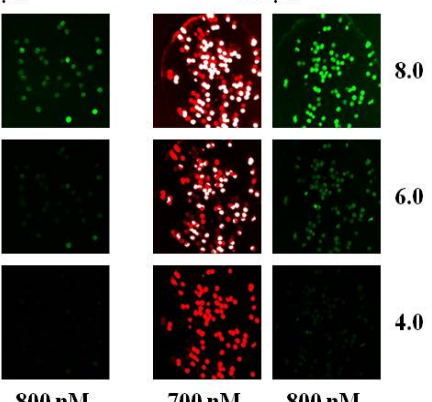

Fig. 2. NIRF of glutaraldehyde-treated different particle sizes of

PAA beads. (a) Beads imaging with fluorescence microscope.

Beads were imaged at the bright field and three different

fluorescence channels, green, red and blue fluorescence.

Magnification: $10 \times 4$. (b) NIRF of different particle sizes of

PAA beads at the emission wavelength of both $700 \mathrm{~nm}$ and 800 $\mathrm{nm}$.

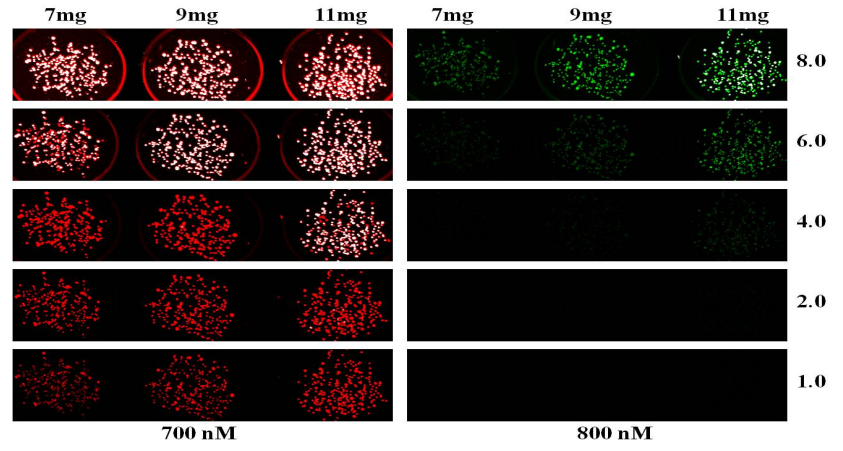

Fig. 3. NIRF of glutaraldehyde-treated PAA beads containing different concentrations of APMA.

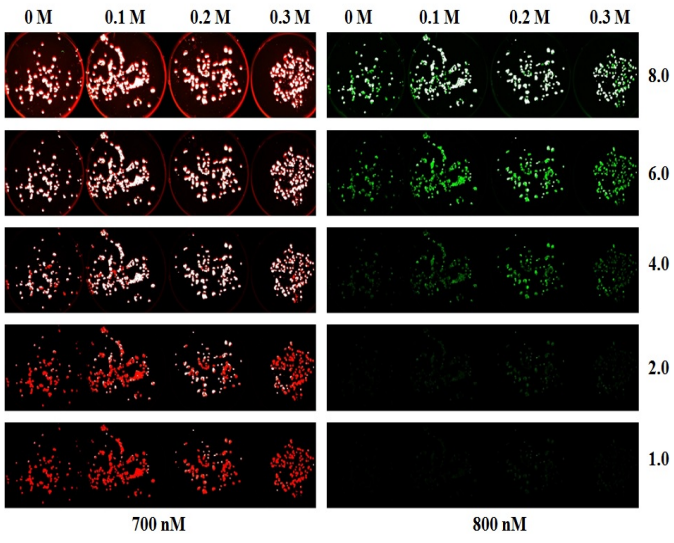

Fig. 4. NIRF of different concentrations of glycine treatment on PAA beads after treated by glutaraldehyde.

compared with the beads containing $7 \mathrm{mg} / \mathrm{mL}$ or $9 \mathrm{mg} / \mathrm{mL}$ APMA in monomer liquid (Fig.3), indicating that the higher the concentration of APMA in beads, the more beneficial it can be to the enhancement of the fluorescence intensity to a certain extent.

Because of the excess of glutaraldehyde in the reaction of polyacrylamide and glutaraldehyde, when beads is soaked in glycine solution, the amino groups on the glycine can continue to react with excess aldehyde on the beads to produce $\mathrm{C}=\mathrm{N}$ bonds in order to enhance the fluorescence intensity. The NIRF imaging results revealed that when glycine solution is $0.2 \mathrm{M}$, the enhancement effect of fluorescence intensity is most obvious (Fig.4). 


\section{Summary}

This paper studied the preparation of fluorescent PAA gel and fluorescent PAA beads on the basis of the autofluorescence at the emission wavelength of both $700 \mathrm{~nm}$ and $800 \mathrm{~nm}$ produced by the reaction between the amino groups and the aldehyde groups. The NIRF imaging results indicate that smaller particle size of PAA beads and higher concentration APMA had stronger fluorescence in a certain range. The fluorescence intensity was significantly enhanced in $0.2 \mathrm{M}$ glycine solution. The most suitable size of PAA beads and the optimum concentration of APMA might be explored based on different applications in further.

\section{Acknowledgement}

This research was financially supported by the National Natural Science Foundation of China (Grant NO. 61571119).

\section{References}

[1] R Zolfaghari, et al., Preparation and characterization of nanocomposite hydrogels based on polyacrylamide for enhanced oil recovery applications, Journal of Applied Polymer Science, vol.100, pp. 2096-2103, 2006.

[2] Y. W. Wang, et al., Study on the drag reduction performance of ionic polyacrylamide, Liaoning Chemical Industry, vol.46, pp. 1166-1170, 2017.

[3] H Kaşgöz, S Özgümüş and M Orbay, Modified polyacrylamide hydrogels and their application in removal of heavy metal ions, Polymer, vol.44, pp. 1785-1793, 2003.

[4] Z Xu and D Zhao, Application of polymer flocculant in soy when effluent treatment, Journal of Harbin University of Commerce, vol. 32, pp. 669-671, 2016.

[5] M. M. Fitzgerald, et al., Tunable stress relaxation behavior of an alginate-polyacrylamide hydrogel: comparison with muscle tissue, Biomacromolecules, vol. 16, pp. 1497-1505, 2015.

[6] T Shirakura, et al., Hydrogel nanoparticles with thermally controlled drug release, ACS Macro Lett, vol. 3, pp. 602-606, 2014.

[7] H. M. Xie, et al., Polyacrylamide nanoparticles with visible and near-infrared autofluorescence, Particle \& Particle Systems Characterization, vol. 34, pp. 1700222, 2017.

[8] M. H. Shapero, et al., SNP genotyping by multiplexed solid-phase amplification and fluorescent minisequencing, Genome Res, vol. 11, pp. 1926-1934, 2001.

[9] A Bellelli, et al., Human erythrocytes cross-linked with glutaraldehyde general properties and significance as a blood substitute, Biochem Biophys Res Commun, vol. 156, pp. 970-977, 1988.

[10]L Rodríguez-Lorenzo, et al., Plasmonic nanosensors with inverse sensitivity by means of enzyme-guided crystal growth, Nature materials, vol.11, pp. 604-607, 2012.

[11]J. S. Collins and T. H. Goldsmith, Spectral properties of fluorescence induced by glutaraldehyde fixation, The Journal of Histochemistry and Cytochemistry, vol. 29, pp.411-414, 1981.

[12]S. Seiffert and W. Oppermann, Amine-functionalized polyacrylamide for labeling and crosslinking purposes, Macromol. Chem. Phys, vol. 208, pp. 1744-1752, 2007. 